\title{
Peristaltic Blood Flow with Gold Nanoparticles on a Carreau Nanofluid through a Non-Darcian Porous Medium
}

\author{
N. T. Eldabe1, G. M. Moatimid1' A. A. El-Shekhipy², Naglaa F. Aballah' ${ }^{1}$ \\ ${ }^{1}$ Department of Mathematics, Faculty of Education, Ain Shams University, Cairo, Egypt \\ ${ }^{2}$ Mathematics Department, Faculty of Science, Imam Abdulrahman Bin Faisal University, Al-Dammam, KSA \\ Email:dr_nagla_1@yahoo.com
}

How to cite this paper: Eldabe, N.T., Moatimid, G.M., El-Shekhipy, A.A. and Aballah, N.F. (2018) Peristaltic Blood Flow with Gold Nanoparticles on a Carreau Nanofluid through a Non-Darcian Porous Medium. Journal of Biomaterials and Nanobiotechnology, 9, 290-306.

https://doi.org/10.4236/jbnb.2018.94017

Received: May 21, 2018

Accepted: October 5, 2018

Published: October 8, 2018

Copyright $\odot 2018$ by authors and Scientific Research Publishing Inc. This work is licensed under the Creative Commons Attribution International License (CC BY 4.0).

http://creativecommons.org/licenses/by/4.0/

\begin{abstract}
The article investigates the influences of a variable thermal conductivity and wall slip on a peristaltic motion of Carreau nanofluid. The model is concerned with heat and mass transfer inside asymmetric channel. The blood is considered as the base Carreau non-Newtonian fluid and gold $\mathrm{(Au}$ ) as nanoparticles stressed upon. The Fronchiener effect of the non-Darcian medium is taken in consideration. The system is stressed upon a strong magnetic field and the Hall currents are completed. The problem is modulated mathematically by a system of non-linear partial differential equations which describe the fluid velocity, temperature and concentration. The system is reformulated under the approximation of long wavelength and low Reynolds number. It is solved on using multi-step differential transform method (Ms-DTM) as a semi-analytical method. A gold nanoparticle has increased the temperature distribution which is of great importance in destroying the cancer cells.
\end{abstract}

\section{Keywords}

Gold Nanoparticles, Peristaltic Blood Flow, Carreau Nanofluid, Non-Darcy Porous Medium, Variable Thermal Conductivity, Multi-Step Differential

Transform Method

\section{Introduction}

Cancer is a dangerous and deadly to most of its patients. Recent studies have shown that gold nanoparticles (GNP) can cure and overcome it because these particles have high atomic numbers which produce heat and leads to treatment of malignancy tumors. GNP has many properties that are very essential in can- 
cer therapy. They are very small and can penetrate widely throughout a body. They have been considered as major area of research due to their applications in biomedical sciences [1]. Huang and El-Sayed [2] studied an important optical and photothermal property of gold nanoparticles in different shapes and structures and address some recent applications for cancer imaging, spectroscopic detection and photothermal therapy. The flow of GNP through two coaxial tubes has been investigated by some authors. Hamzehnezhad et al. [3] and Hatami et al. [4] studied the third-grade non-Newtonian fluid conveying GNP in a porous and hollow vessel by various analytical methods which is essential idea of the present paper.

The studying is that Darcian and non-Darcian flow in Newtonian and non-Newtonian fluid flow saturating porous medium. This because of their wide applications such as chemical reactors, building non-Darcian fluids in saturated porous media meets a great importance. The Dag insulation, packed bed, enhanced oil recovery, food technology and filtration processes. Eldabe and Abu Zeid [5] have studied a non-Darcian Couette flow through a porous medium of magnetohydrodynamic visco-elastic fluid with heat and mass transfer. They found the solutions of velocity, temperature and nanoparticles distribution by using the homotopy perturbation method. The effect of the boundary conditions on a peristaltic flow of a nanofluid in an asymmetric channel embedded with a non-Darcian porous medium was scrutinized by Rathod and Sanjeevkumar [6]. Wu et al. [7] studied the analysis of multiphase Non-Darcian flow in porous media.

In the literature, numerous studies regarding peristaltic flow of a Carreau nanofluid have been done for Non-Newtonian fluids. Akbar et al. [8] studied the numerical simulation of peristaltic flow of a Carreau nanofluid in an asymmetric channel. They found solutions for the distributions of stream function, pressure rise, and temperature and nanoparticle volume fraction by using the fourth and fifth order Runge-Kutta-Fehlberg. The effects of Nanofluid on peristaltic flow of a Carreau fluid model in an inclined magnetic field are proposed by Akram [9]. He found the solutions of the simplified coupled nonlinear equations using an analytical approach. Eldabe et al. [10] scrutinized the peristaltic transport of magnetohydrodynamic Carreau nanofluid with heat and mass transfer inside asymmetric channel.

Peristaltic flow of a nanofluid under the effect of the hall current and porous medium was studied by Nowar [11]. Abo-Eldahab et al. [12] introduced the hall currents and heat transfer effects on peristaltic transport in a vertical asymmetric channel through a porous medium. Steh and Ghosh [13] addressed the combined influence of rotation and Hall current in the presence of a transverse magnetic field, neglecting the induced magnetic field. Ghosh [14] discussed the effects of Hall current on an unsteady hydromagnetic flow in a rotating channel permeated by an inclined magnetic field in the presence of an oscillator.

Several applications in engineering occur at high temperature through variable thermal conductivity. Few such operations include nuclear power plants, in 
turbines, rockets, space vehicles and pumps operated at high temperatures (see Ref. [15] [16]). In addition, studying velocity slip effects are adequate for certain flow problems at the walls of the pipes/channels.

The governing equations of motion become more complex to handle as supplemental nonlinear terms. So, the exact solutions of such problems are practically impossible, so we turn to find a new method such as the differential transform method (DTM). DTM is semi-analytical methods appear in the equations of motion, because of the flow behavior of Non-Newtonian nanofluids. This method was proposed by Zhou [17], for solving ordinary, partial differential and integral equations. Different applications of DTM can be found in [18]. The Ms-DTM method accelerates the convergence of the series solution over large region and yields a series solution, this series will be truncated due to the required accuracy of solutions. This modified technique is verified through illustrative examples of non-chaotic or chaotic systems by Odibat et al. [19].

To the best of our knowledge, the effect of the Hall current, variable thermal conductivity on peristaltic flow of Carreau nanofluid has not been discussed before. Motivated by the above discussions, the aim of the present paper is to examine the MHD peristaltic flow of Carreau nanofluid accompanying heat and mass transfer in the presence of viscous dissipation. In order to clarify the problem at hand, the mathematical modeling is affirmed in Section 2, solutions of the problem on using the multi-steps DTM are presented in Section 3. In Section 4 the influence of the various parameters, the pressure gradient, pressure rise, longitudinal velocity, temperature and concentration are explored through figures and tables. The main findings have been abbreviated in Section 5.

\section{Formulation of the Problem}

Consider a peristaltic flow of an incompressible Carreau nanofluid in a two dimensional asymmetric channel of width $d_{1}+d_{2}$. It is convenient to work with the Cartesian coordinates $(X, Y)$ where $X$-axis and $Y$-axis are the horizontal and vertical axes, respectively. The right and left walls have a constant temperature of $T_{1}$ and $T_{0}$, respectively. Also, they have nanoparticle volume fractions of $C_{1}$ and $C_{0}$. Asymmetry in the channel is produced by propagation of waves along the channel walls and traveling with different amplitudes, phases and of the constant speed. The sketch of the model is given in Figure 1. In the Cartesian coordinates system $(X, Y)$, the right wall $Y=H_{1}$ and the left wall $Y=H_{2}$. They are given from the following relations:

$$
\begin{gathered}
Y=H_{1}=d_{1}+a_{1} \cos \left[\frac{2 \pi}{\lambda}(X-c t)\right] \quad \text { Right hand side } \\
Y=H_{2}=-d_{2}-b_{1} \cos \left[\frac{2 \pi}{\lambda}(X-c t)+\phi\right] \quad \text { Left hand side }
\end{gathered}
$$

where $a_{1}$ and $b_{1}$ are the amplitudes of the waves, $\lambda$ is the wave length, the phase difference $\phi$ diverse in the range $(0 \leq \phi \leq \pi), \phi=0$, corresponds to 


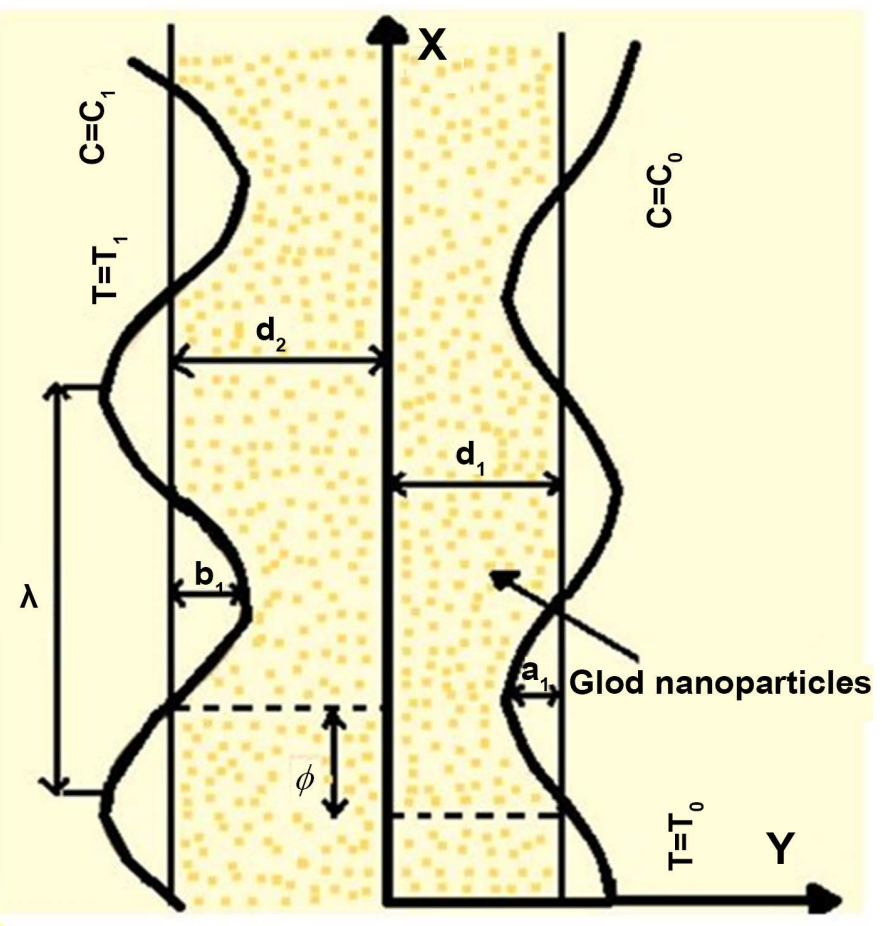

Figure 1. Geometry of physical model.

symmetric channel with waves out of phase and $\phi=\pi$ the waves are in phase, and further $a_{1}, b_{1}, d_{1}, d_{2}$ and satisfies the condition.

$$
a_{1}^{2}+b_{1}^{2}+2 a_{1} b_{1} \cos \phi \leq\left(d_{1}^{2}+d_{2}^{2}\right)
$$

A strong uniform magnetic field with magnetic flux density $B=\left(0,0, B_{0}\right)$ is applied and the Hall effects are taken, also into account. Assuming that no applied polarization voltage, so that the electric field ignored. In case of the hall current, as given from generalization of the ohm [20]:

$$
\bar{J}=\sigma\left[E+\bar{V} \times \bar{B}-\frac{1}{e\left(n_{e}\right)}(\bar{J} \times \bar{B})\right],
$$

where $\sigma$ is the electrical conductivity of the fluid, $\bar{V}$ is the velocity vector, $e$ is the electric charge of electrons, $n_{e}$ is the number density of the electrons. Equation (3) can be solved in $\bar{J}$ to yield the Lorentz force vector in the form:

$$
\bar{J} \times \bar{B}=\frac{\sigma B_{0}^{2}}{1+m^{2}}[(V+m U) \underline{i}+(m V-U) \underline{j}],
$$

where $U$ and $V$ are the $X$ and $Y$ components of the velocity vector $V=\left(V_{x}, V_{y}, 0\right)$, $m=\frac{\sigma B_{0}}{e\left(n_{e}\right)}$ is the hall parameter.

The variable thermal conductivity, with dimensionless temperature $\theta$ is taken into account where,

$$
k(\theta)=k_{0}(1+\epsilon \theta), \text { for } \epsilon<1
$$


Here $\epsilon=\left(T_{m}-T_{w}\right)$ is the thermal conductivity variation parameter, $k_{0}$ is the thermal conductivity constant parameter at $\epsilon=0$.

The constitutive equation for a Carreau fluid is given by [8].

$$
\frac{\eta-\eta_{\infty}}{\eta_{0}-\eta_{\infty}}=\left[1+(\Gamma \dot{\gamma})^{2}\right]
$$

and

$$
\tau_{i j}=\eta_{0}\left[1+\frac{n-1}{2}(\Gamma \dot{\gamma})^{2}\right] \dot{\gamma}_{i j},
$$

In which $\tau_{i j}$ is the extra stress tensor, $\eta_{\infty}$ is the infinite shear rate viscosity, $\eta_{0}$ is the zero shear rate viscosity, $\Gamma$ is the time constant, $n$ is the power law index, and $\dot{\gamma}$ is defined as:

$$
\dot{\gamma}=\sqrt{\frac{1}{2} \sum_{i} \sum_{j} \dot{\gamma}_{i j} \dot{\gamma}_{j i}}=\sqrt{\frac{1}{2} \Pi} .
$$

where $\prod .=\operatorname{trac}\left(\operatorname{grad} V+(\operatorname{grad} V)^{\mathrm{T}}\right)^{2}$ is the second invariant strain tensor. For considering the natural convection, the nanofluid density $\rho$ is defined by [2] as:

$$
\rho=\varphi \rho_{p}+(1+\varphi) \rho_{f_{0}} \cong \varphi \rho_{p}+(1+\varphi)\left[\rho_{f}\left(1-\beta_{T}\left(\vartheta-\theta_{w}\right)\right)\right],
$$

where $\rho_{p}$ is the density of the gold nanoparticles, $\rho_{f_{0}}$ is the base fluid's density, $\vartheta$ is the temperature distribution, $\theta_{w}$ is the reference temperature, $\beta_{T}$ is the volumetric coefficient of the expansion and $\rho_{f}$ is the nanofluid's density at the reference temperature, $\varphi$ is the concentration of nanoparticles.

The governing equations of peristaltic flow in the case of an incompressible flow of Carreau nanofluid are given by [4] [8]:

The continuity equation:

$$
\frac{\partial U}{\partial X}+\frac{\partial V}{\partial Y}=0
$$

The conversation of momentum yields

$$
\begin{gathered}
\rho_{f}\left[\frac{\partial U}{\partial t}+U \frac{\partial U}{\partial X}+V \frac{\partial U}{\partial Y}\right] \\
=-\frac{\partial P}{\partial X}+\frac{\partial}{\partial X}\left(\tau_{X X}\right)+\frac{\partial}{\partial Y}\left(\tau_{X Y}\right)-\frac{\sigma B_{0}^{2}}{1+m^{2}}(V+m U)+\varphi \rho_{p} \\
+(1+\varphi)\left[\rho_{f}\left(1-\beta_{T}\left(\vartheta-\theta_{w}\right)\right)\right] g-\frac{\eta_{0}}{k_{1}}-\frac{\rho_{f} C_{b}}{\sqrt{k_{1}}} U^{2}, \\
\rho_{f}\left[\frac{\partial V}{\partial t}+U \frac{\partial V}{\partial X}+V \frac{\partial V}{\partial Y}\right] \\
=-\frac{\partial P}{\partial Y}+\frac{\partial}{\partial X}\left(\tau_{Y X}\right)+\frac{\partial}{\partial Y}\left(\tau_{Y Y}\right)+\frac{\sigma B_{0}^{2}}{1+m^{2}}(m V-U)-\frac{\mu_{f}}{k_{1}} V-\frac{\rho_{f} C_{b}}{\sqrt{k_{1}}} V^{2},
\end{gathered}
$$


The energy equation gives

$$
\begin{aligned}
& (\rho c)_{f}\left[\frac{\partial T}{\partial t}+U \frac{\partial T}{\partial X}+V \frac{\partial T}{\partial Y}\right] \\
& =\frac{\partial}{\partial X}\left(k(T) \frac{\partial T}{\partial X}\right)+\frac{\partial}{\partial Y}\left(k(T) \frac{\partial T}{\partial y}\right)+\frac{\sigma B_{0}^{2}}{1+m^{2}}\left(U^{2}+V^{2}\right) \\
& +\left[\tau_{X X} \frac{\partial U}{\partial X}+\tau_{X Y} \frac{\partial U}{\partial Y}+\tau_{Y X} \frac{\partial V}{\partial Y}+\tau_{Y Y} \frac{\partial V}{\partial Y}\right] \\
& +(\rho c)_{p}\left[\frac{D_{T}}{T_{0}}\left[\frac{\partial^{2} T}{\partial X^{2}}+\frac{\partial^{2} T}{\partial Y^{2}}\right]+D_{B}\left(\frac{\partial C}{\partial X} \frac{\partial T}{\partial X}+\frac{\partial C}{\partial Y} \frac{\partial T}{\partial Y}\right)\right],
\end{aligned}
$$

The concentration equation gives

$$
\left[\frac{\partial C}{\partial t}+U \frac{\partial C}{\partial X}+V \frac{\partial C}{\partial Y}\right]=D_{B}\left(\frac{\partial^{2} C}{\partial X^{2}}+\frac{\partial^{2} C}{\partial Y^{2}}\right)+\frac{D_{T}}{T_{0}}\left[\frac{\partial^{2} T}{\partial X^{2}}+\frac{\partial^{2} T}{\partial Y^{2}}\right] .
$$

where $P$ is the pressure, $k_{1}$ is the permeability of the porous medium, $g$ is acceleration of the gravity, $T$ is the temperature of the fluid and $C$ is the nanoparticles concentration. Both the magnetic field and channel are inclined at angle, $(\rho c)_{f}$ is the heat capacity of the fluid, $k$ is the thermal conductivity, (c) $p$ is the effective heat capacity of the nanoparticles material, $D_{T}$ is the thermophoretic diffusion and $D_{B}$ is the Brownian diffusion coefficient. Using similar arguments as given by [2] [8], we may assume the following transformations, $x=X-c t, y=Y, u=U-c, v=V$ and $p(x)=P(X, t)$.

To simplify the equations of motion, we may consider the following dimensionless quantities:

$$
\begin{aligned}
& \bar{x}=\frac{x}{\lambda}, \bar{y}=\frac{y}{d_{1}}, \bar{u}=\frac{u}{c}, \bar{v}=\frac{v}{c \delta}, \bar{p}=\frac{d_{1}^{2}}{\mu c \lambda}, h_{1}=\frac{H_{1}}{d_{1}}, h_{2}=\frac{H_{2}}{d_{2}}, d=\frac{d_{2}}{d_{1}}, a=\frac{a_{1}}{d_{1}} \\
& b=\frac{b_{1}}{d_{1}}, R_{e}=\frac{\rho_{f} c d_{1}}{\eta_{0}}, \bar{\psi}=\frac{\psi}{c d_{1}}, \bar{\tau}_{x x}=\frac{\lambda}{\eta_{0} c} \tau_{x x}, \bar{\tau}_{x y}=\frac{d_{1}}{\eta_{0} c} \tau_{x y}, \bar{\tau}_{y y}=\frac{d_{1}}{\eta_{0} c} \tau_{y y}, \\
& W_{e}=\frac{\Gamma c}{d_{1}}, \overline{\dot{\gamma}}=\frac{d_{1} \dot{\gamma}}{c}, s=\frac{k_{1}}{d_{1}^{2}}, \alpha=\frac{K}{(\rho c)_{f}}, \theta=\frac{\theta^{\prime}-\theta_{w}}{\theta_{m}-\theta_{w}}, \varphi=\frac{\varphi^{\prime}-\varphi_{w}}{\varphi_{m}-\varphi_{w}}, P_{r}=\frac{\eta_{0}}{\alpha \rho_{f}}, \\
& G_{r}=\frac{\rho_{f} d_{1}^{2} g\left(\theta_{m}-\theta_{w}\right)\left(1-\varphi_{m}\right)}{c \eta_{0}}, B_{r}=\frac{a_{1}^{2} \rho\left(\rho_{p}-\rho_{f} g\right)\left(\varphi_{m}-\varphi_{w}\right)}{c \eta_{0}}, \\
& N_{b}=D_{B}\left(\varphi_{m}-\varphi_{w}\right), N_{t}=\frac{D_{T}\left(\theta_{m}-\theta_{w}\right)}{\theta_{w}}, C_{f}=\frac{\rho_{f} C_{b} d_{1}^{2} c}{\eta_{0} \sqrt{K}} .
\end{aligned}
$$

where $\delta$ is the dimensionless wave number, $R_{e}$ is the Reynolds number, $P_{r}$ is the Prandtl number, $C_{f}$ is the Forchheimer number, $G_{r}$ is the local Grashof number, $B_{r}$ is the local nanoparticles Grash of number, $N_{b}$ is the Brownian motion parameter and $N_{t}$ is the thermophoresis parameter, $W_{e}$ is the Weissenberg number, $M$ is the magnetic parameter. Here $\theta_{m}$ and $\varphi_{m}$, denote the pipe temperature, fluid temperature and mass concentration, respectively. As usual in fluid mechanics, the stream function $\psi=\psi(x, y)$ may be taken as $u=\frac{\partial \psi}{\partial y}$ and $v=-\frac{\partial \psi}{\partial x}$. 


$$
\tau_{x y}=\left[1+\frac{n-1}{2} W_{e}^{2} \dot{\gamma}^{2}\right]\left(\frac{\partial^{2} \psi}{\partial y^{2}}-\delta^{2} \frac{\partial^{2} \psi}{\partial x^{2}}\right)=\tau_{y x},
$$

The governing equations of the Carreau nanofluid are:

$$
\begin{aligned}
& -\frac{\partial p}{\partial x}+\frac{\partial}{\partial y}\left[1+\frac{n-1}{2} W_{e}^{2}\left(\frac{\partial^{2} \psi}{\partial y^{2}}\right)^{2}\right] \frac{\partial^{2} \psi}{\partial y^{2}}-\left(\frac{M^{2}}{1+m^{2}}+2 C_{f}\right) \frac{\partial \psi}{\partial y} \\
& -C_{f} \frac{\partial^{2} \psi}{\partial y^{2}}+G_{r} \theta+B_{r} \varphi=0, \\
& -\frac{\partial p}{\partial y}=0, \\
& \frac{1}{P_{r}}\left(\epsilon\left(\frac{\partial \theta}{\partial y}\right)^{2}+\left(1+\epsilon \frac{\partial^{2} \theta}{\partial y^{2}}\right)\right)+E_{c}\left[\frac{\partial^{2} \psi}{\partial y^{2}}+\frac{n-1}{2} W_{e}^{2}\left(\frac{\partial^{2} \psi}{\partial y^{2}}\right)^{4}\right] \\
& +E_{c} \frac{M^{2}}{1+m^{2}}\left(\frac{\partial \psi}{\partial y}+1\right)^{2}+\frac{N_{b}}{P_{r}} \frac{\partial \theta}{\partial y} \frac{\partial \varphi}{\partial y}+\frac{N_{t}}{P_{r}}\left(\frac{\partial \theta}{\partial y}\right)^{2}=0, \\
& \frac{\partial^{2} \varphi}{\partial y^{2}}+\frac{N_{t}}{N_{b}} \frac{\partial^{2} \theta}{\partial y^{2}}=0 .
\end{aligned}
$$

The eliminating of the pressure from Equations (17) and (18) gives

$$
\begin{aligned}
& \frac{\partial^{2}}{\partial y^{2}}\left[1+\frac{n-1}{2} W_{e}^{2}\left(\frac{\partial^{2} \psi}{\partial y^{2}}\right)^{2}\right] \frac{\partial^{2} \psi}{\partial y^{2}}-\left(\frac{M^{2}}{1+m^{2}}+2 C_{f}\right) \frac{\partial^{2} \psi}{\partial y^{2}} \\
& -C_{f} \frac{\partial^{3} \psi}{\partial y^{3}}+G_{r} \frac{\partial \theta}{\partial y}+B_{r} \frac{\partial \varphi}{\partial y}=0
\end{aligned}
$$

The appropriate boundary conditions may be represented as:

$$
\begin{aligned}
& \psi=\frac{q}{2}, \theta=0, \varphi=0, \frac{\partial \psi}{\partial y}-L \frac{\partial^{2} \psi}{\partial y^{2}}=-1, \text { at } y=h_{1}=1+a \cos 2 \pi x, \\
& \psi=\frac{-q}{2}, \theta=0, \varphi=0, \frac{\partial \psi}{\partial y}=-1, \text { at } y=h_{2}=-d-b \cos (2 \pi x+\phi),
\end{aligned}
$$

The dimensional time mean flow rate $Q$ in the laboratory frame is related to $q$ through the relation [20] [21]

$$
Q=q+1+d
$$

Now, the system of nonlinear differential Equations (19)-(21) subjected to the appropriate boundary conditions (22)-(32) must be solved, thus we use the Ms-DTM [18] [19].

\section{The Method of Solution}

The above system may be solved by the MS-DTM method as follows. Using similar arguments as given by [19], the functions $\psi(y), \theta(y)$ and $\varphi(y)$ are given from the following Equations. 


$$
\begin{aligned}
& \quad[k+1][k+2][k+3][k+4] \Psi[k+4] \\
& +\frac{1}{2}(-1+n) W_{e}^{2} \sum_{r=0}^{k}\left[\begin{array}{c}
{[k+1][k-r+1][k-r+2][k-r+3]} \\
{[k-r+4] \Psi[r+1] \Psi[k-r+2] \Psi[k-r+4]}
\end{array}\right] \\
& \quad-\left(\frac{M^{2}}{1+m^{2}}+\frac{1}{S}\right) \sum_{r=0}^{k}[r+1][k-r+1] \Psi[r+1] \Psi[k-r+1] \\
& +G_{r}[k+1] \Theta[k+1]+B_{r}[k+1] \Phi[k+1] \\
& \quad+3(-1+n) W_{e}^{2}\left(\sum_{r=0}^{k}[r+1][k-r+1][k-r+2][k-r+3]\right. \\
& \quad \times \Psi[r+1] \Psi[k-r+2] \Psi[k-r+3])+\sum_{r=0}^{k}[r+1][k-r+1][k-r+2] \\
& \quad \times[k-r+3][k-r+4] \Psi[k-r+2] \Psi[k-r+4] \\
& \quad-2 C_{f}[k+1][k+2] \Psi[k+2]-C_{f}[k+1][k+2][k+3] \Psi[k+3], \\
& \frac{\epsilon}{P_{r}}\left[\sum_{r=0}^{k}[r+1][k-r+1] \Theta[r+1] \Theta[k-r+1]\right. \\
& +\frac{\epsilon}{P_{r}} \sum_{r=0}^{k}[[r+1][k-r+1][k-r+2]] \\
& \left.+\frac{N_{b}}{P_{r}} \sum_{r=0}^{k}[r+1][k-r+1][k-r+2] \Phi[k-r+2] \Theta[k-r+1]\right] \\
& +\frac{N_{t}}{P_{r}} \sum_{r=0}^{k}[r+1][k-r+1] \Theta[r+1] \Theta[k-r+1] \\
& +E_{c} \frac{M^{2}}{1+m^{2}}\left[1+2[k+1] \Psi[k+1]+\sum_{r=0}^{k}[r+1][k-r+1] \Psi[r+1] \Psi[k-r+1]\right. \\
& +[k+1][k+2] \Psi[k+2]]+\frac{1}{P_{r}}[k+1][k+2] \Theta[k+2] \\
& +E_{c} \frac{n-1}{2} W_{e}^{2} \sum_{r=0}^{k}[k+1][k+2] \Psi[k+2]+[r+1][k-r+1] \\
& \times[k-r+2][k-r+3][k-r+4] \Psi[r+1] \Psi[k-r+4], \\
& \quad[k+1][k+2] \Phi[k+2]+\frac{N_{t}}{N_{b}}[k+1][k+2] \Theta[k+2]=0 \\
&
\end{aligned}
$$

where $\Psi[k], \Theta[k]$ and $\Phi[k]$ are the differential transformation functions of $\psi(y), \theta(y)$ and $\varphi(y)$ respectively. The differential transform of the associated boundary conditions are given by:

$$
\begin{aligned}
& \left.\Psi_{0}=-\frac{q}{2}, \Psi_{1}-2 L \Psi_{2}=-1, \Theta_{0}=1, \Phi_{0}=1 \text { at } y=h_{2}(x)=-d-b \cos (2 \pi x+\phi),(27)\right]^{k}=\frac{q}{2} \\
& \sum_{k=0}^{n} \Psi_{k}\left[h_{1}(x)-h_{2}(x)\right. \\
& \sum_{k=1}^{n} k \Psi_{k}\left[h_{1}(x)-h_{2}(x)\right]^{k-1}-L \sum_{k=1}^{n} k(k-1) \Psi_{k}\left[h_{1}(x)-h_{2}(x)\right]^{k-1}=-1, \\
& \sum_{k=0}^{n} \Theta_{k}\left[h_{1}(x)-h_{2}(x)\right]^{k}=0, \\
& \sum_{k=0}^{n} \Phi_{k}\left[h_{1}(x)-h_{2}(x)\right]^{k}=0, \text { at } y=h_{1}(x)=1+a \cos (2 \pi) .
\end{aligned}
$$

The expansions of the distributions of $\psi(y), \theta(y)$ and $\varphi(y)$ up to the 
tenth-order may be listed as follows:

$$
\begin{aligned}
\psi(y)= & 0.75-1.00429 y-0.0428583 y^{2}+0.196714 y^{3} \\
& +0.09676565239842302 y^{4}+0.001193861687515796 y^{5} \\
& -0.0006033224257142588 y^{6}-0.0022550902628023194 y^{7} \\
& +0.0004527902505426113 y^{8}+0.0003671545327072134 y^{9} \\
& -0.0000736874408943607 y^{10} \\
\theta(y)= & 0.807854 y-2.5917363287244672 y^{2}+2.2974739187465896 y^{3} \\
- & 1.5353449243714945 y^{4}+0.7069612997113166 y^{5} \\
- & 0.27258261651934795 y^{6}+0.11458745801237043 y^{7} \\
- & 0.05626101276801457 y^{8}+0.027201065873438676 y^{9} \\
- & 0.0124587997837748 y^{10}
\end{aligned}
$$

And

$$
\begin{aligned}
\varphi(y)= & -0.00870575 y+4.535538575267818 y^{2}-4.020579357806532 y^{3} \\
& +2.686853617650115 y^{4}-1.237182274494804 y^{5} \\
& +0.47701957890885893 y^{6}-0.20052805152164827 y^{7} \\
& +0.0984567723440255 y^{8}-0.04760186527851768 y^{9} \\
& +0.0218028996216059 y^{10} .
\end{aligned}
$$

\section{Graphical Results and Discussions}

The solutions acquired by the Ms-DTM are displayed through the following numerical calculation. The aim of these calculations is to obtain the influences of various parameters on the distributions for each of pressure gradient and pressure rise as well as velocity, temperature and concentration as the follows:

Figures 2(a)-(e) show the influences of the different parameters on the distribution of pressure gradient $\frac{\mathrm{d} P}{\mathrm{~d} x}$. It is found that the pressure gradient increases with the increase of $M, C_{f}$ and $N_{b}$. Therefore larger pressure gradient is needed for the motivation the flow through the channel. It is a usual sense. Meanwhile, it decreases by increasing $N_{t}$. As seen in Figure $2(\mathrm{e})$ the pressure gradient decreases in $x \in[0.0,0.2989]$ and $x \in[0.544,1.0]$. Meanwhile it increases in $x \in[0.342,0.489]$ with the increase of $G_{r}$, which is named by phenomenon dual role.

Figures 3(a)-(f) show the influences of the different parameters on the distribution of pressure rise $\Delta P$ with volume flow rate $Q$ for different values of $N_{t}, B_{r}, N_{b}, L$ and $G_{r}$ It is seen in Figure 3(a), Figure 3(b) the pressure rise decreases in all pumping regions with an increase in $N_{t}$ and $B_{r}$. Meanwhile the converse behavior was observed with the increment in $N_{b}$ through Figure $3(\mathrm{c})$. Figure 3(d), Figure 3(e) show that pressure rise increases in the retrograde pumping $(\Delta P>0, Q<0)$, free pumping $\left(Q=Q_{0}>0 ; \Delta P=0\right)$ and peristaltic pumping $(\Delta P>0, Q>0)$ regions with an increase in $L$ and $C_{f}$. Meanwhile in the augmented pumping region $(\Delta P<0, Q>0)$ the pressure rise increases 


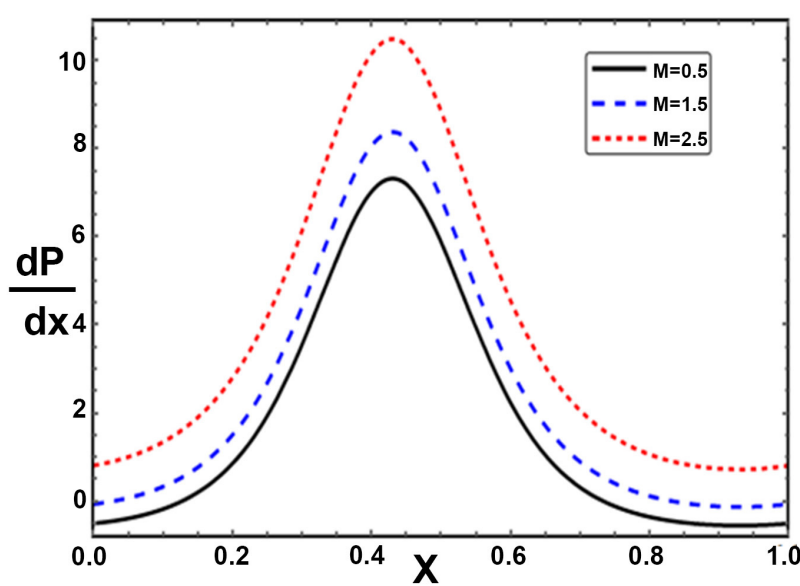

(a)

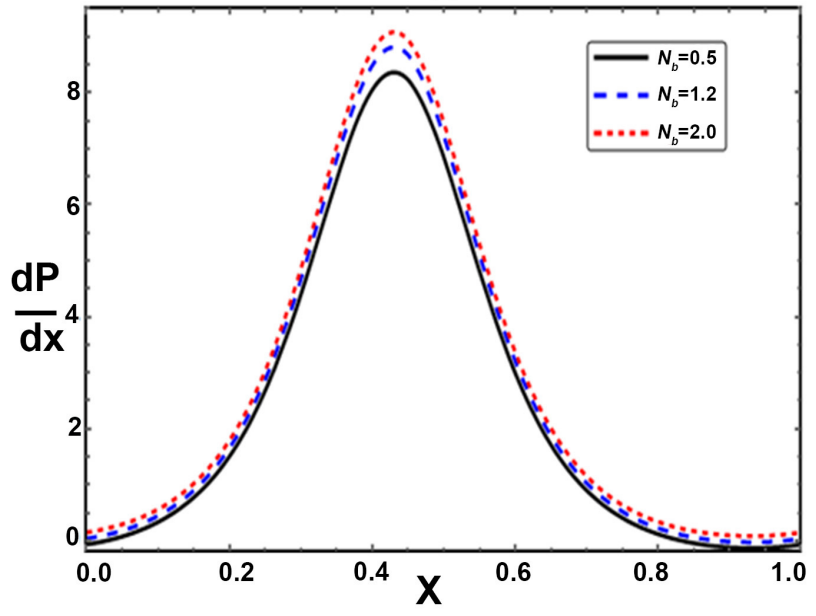

(c)

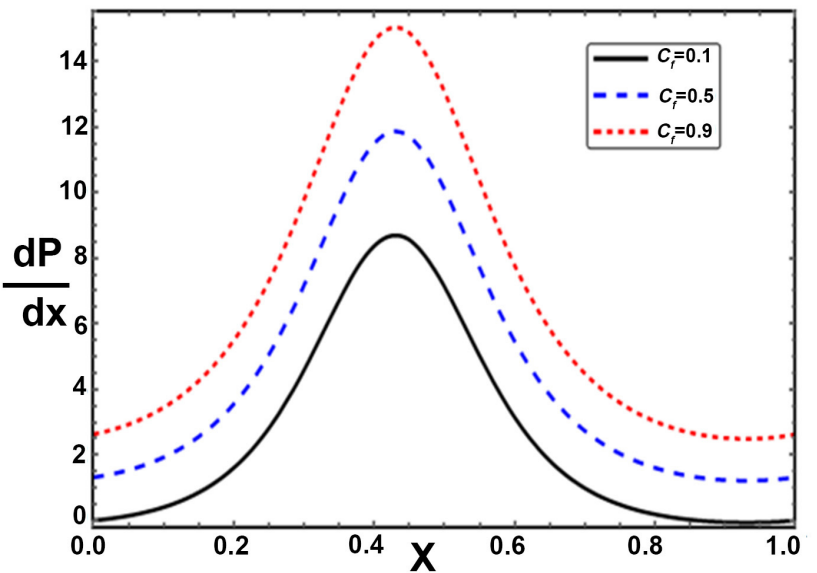

(b)

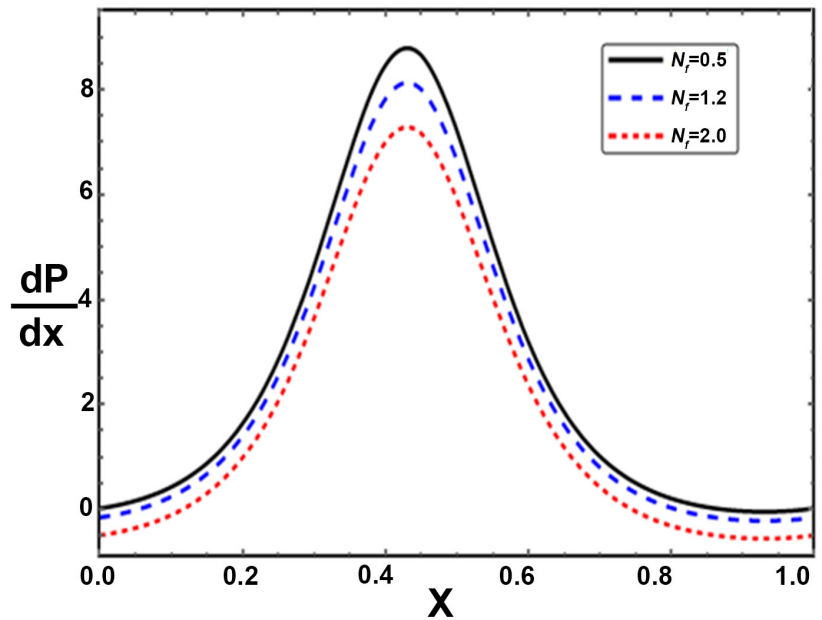

(d)

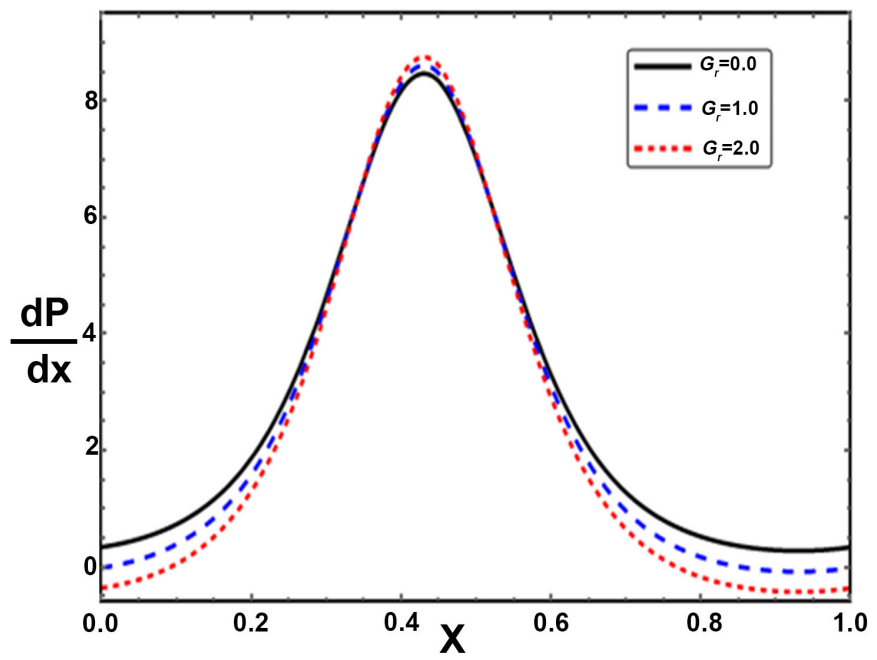

(e)

Figure 2. (a)-(e). Effects of embedded parameters on the pressure gradient, (a) $G_{r}=1, C_{f}=0.5, N_{b}=0.7, N_{t}=0.8 ;(\mathrm{b})$ $G_{r}=1, N_{b}=0.5, M=1, N_{t}=0.8$; (c) $G_{r}=1, C_{f}=0.5, M=1, N_{t}=0.8$; (d) $G_{r}=1, C_{f}=0.5, M=1, N_{b}=0.8 ;(\mathrm{e})$ $N_{b}=0.7, C_{f}=0.5, M=1, N_{t}=0.8$, the other parameters are chosen as $x=0, B_{r}=1, P_{r}=2, q=1, \vartheta=\frac{\pi}{4}, a=0.4$, $b=0.5, d=1.5, W_{e}=0.5, n=1, E_{c}=0.2, \quad m=1, \epsilon=0.1, L=0.1, \alpha=0.2$. 


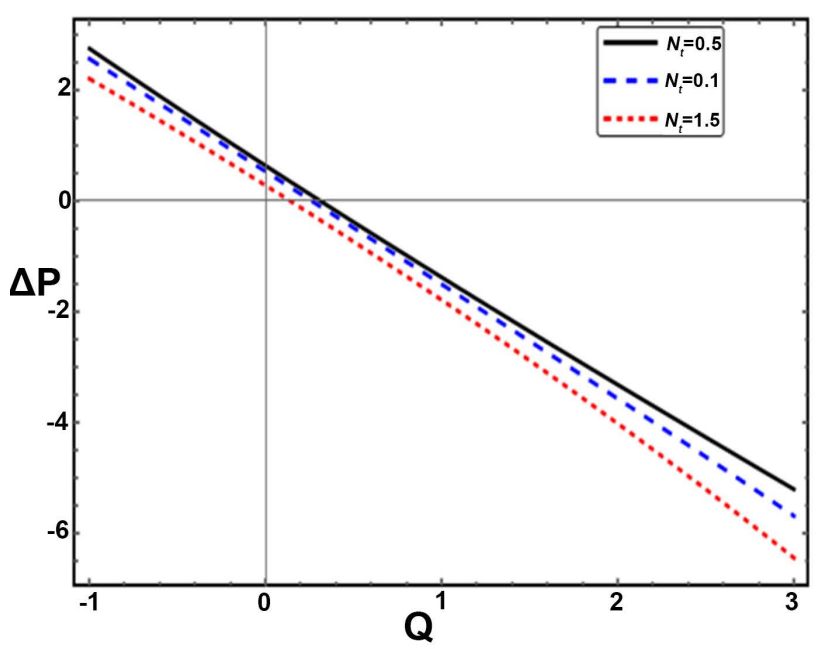

(a)

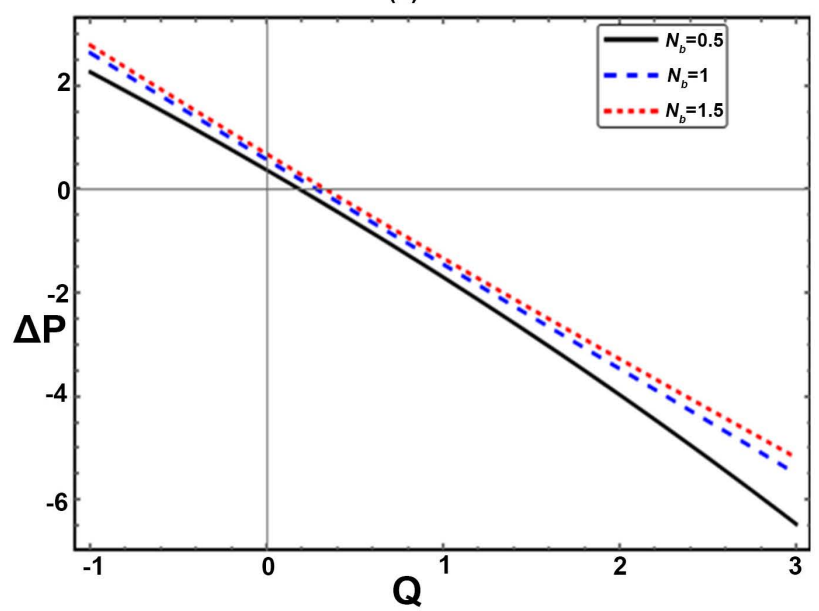

(c)

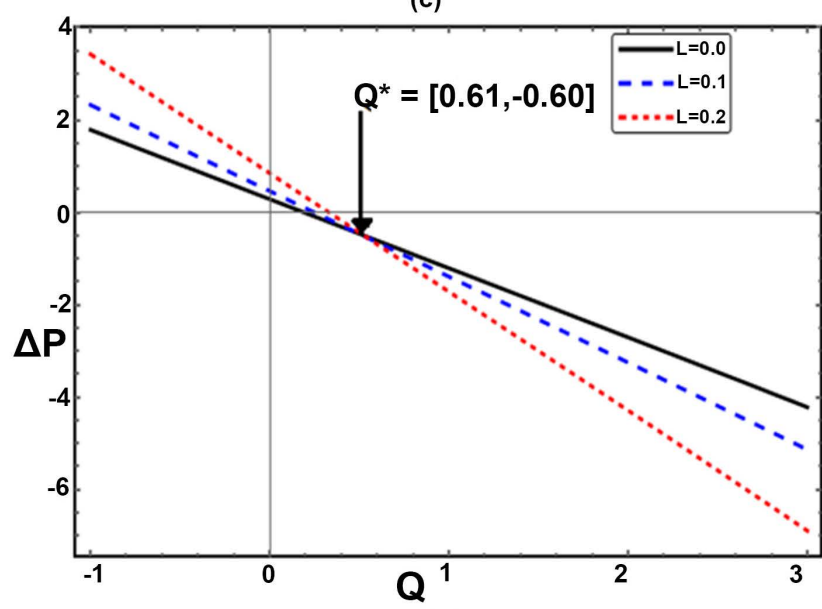

(e)

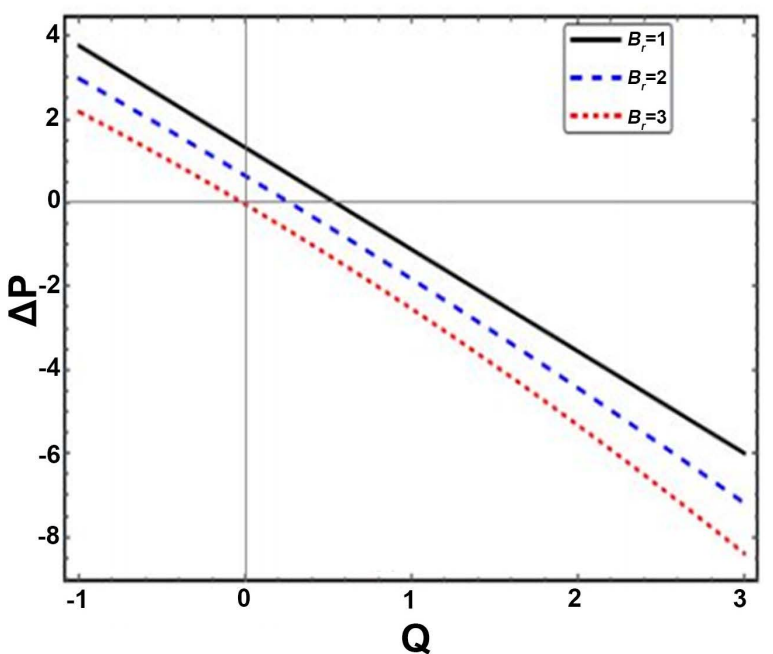

(b)

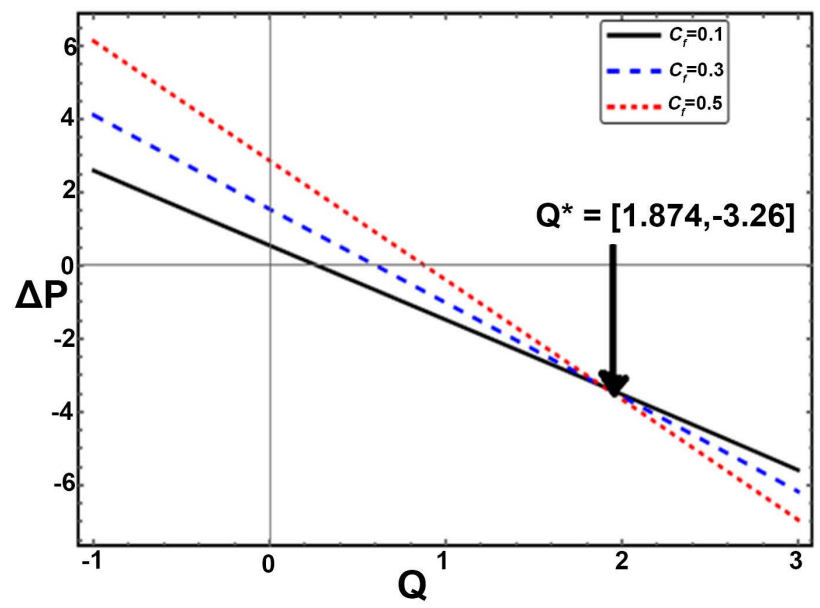

(d)

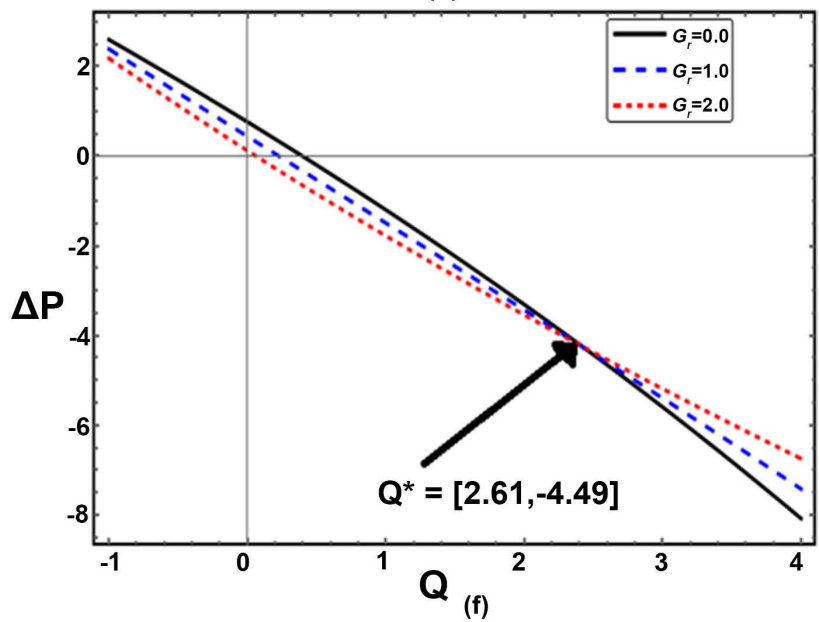

Figure 3. (a)-(f). Effects of embedded parameters on the pressure rise, (a) $B_{r}=1, G_{r}=1, C_{f}=0.5, L=0.1, N_{b}=0.7 ;$ (b) $G_{r}=1, C_{f}=0.5, L=0.1, N_{b}=0.7, N_{t}=0.8$; (c) $B_{r}=1, G_{r}=1, L=0.1, C_{f}=0.7, N_{t}=0.8 ;(\mathrm{d}) \quad B_{r}=1, G_{r}=1$, $L=0.1, N_{b}=0.7, N_{t}=0.8$; (e) $B_{r}=1, G_{r}=1, C_{f}=0.5, N_{b}=0.7, N_{t}=0.8$; (f) $B_{r}=1, C_{f}=0.5, L=0.1, N_{b}=0.7$, $N_{t}=0.8$, the other parameters are chosen as $x=0, P_{r}=2, q=1, \vartheta=\frac{\pi}{4}, a=0.4, b=0.5, d=1.5, W_{e}=0.5, n=1$, $E_{c}=0.2, \quad m=1, \epsilon=0.1, \quad \alpha=0.2$. 
till a certain value $Q$ then it decreases (case of increasing in $C_{f}$ ), also in case of increasing in $L$ the pressure rise increases till a certain value $Q^{*}$ then the pressure rise decreases in the augmented pumping region. Figure 3(f) portrayed that the pressure rise decreases in all pumping regions till a certain value $Q^{*}$ were lies in the augmented pumping region then the pressure rise increases.

Figures 4(a)-(e) show the influences of the different parameters on the distribution of velocity profile. It is seen in Figure 4(a), Figure 4(b) the velocity profile increases at the central of the channel in $y \in[1.201,0.21]$. Meanwhile it decreases at the left and right hand sides of the channel in $y \in[0.22,1.28]$ and $y \in[1.85,1.31]$ with the increase of in $M$ and $C_{f}$. Therefore, these Figs show that the gold nanoparticles are active in drug carrying and drug delivery systems, because they can encapsulate large quantities of therapeutic molecules. Figure 4 (c), Figure 4(d) depicted that the velocity increases in the region $y \in[1.8769,0.6923]$, Meanwhile it decreases at the right hand side in a region $y \in[0.72,0.764]$ of the channel with the increase of $W_{e}$ and $N_{b}$. However the converse behavior can be observed through Figure 4(e) with the increase of $N_{t}$. This shows the dual role of the influence of the parameters on temperature.

Figures 5(a)-(f) show the influences of the different parameters on the distribution of temperature $\theta$ It is seen in Figures 5(a)-(d) the temperature distribution increases with the increase of $W_{e}, N_{b}, N_{t}$ and $M$. Also it is noted that increases of the temperature distribution in the case of gold. Nanoparticles can be of help the treatment of cancer. Figure 5(e), Figure 5(f) portrayed that by increasing variable thermal conductivity parameter $\epsilon$ and Forchheimer number $C_{f}$ the temperature distribution decreases.

Figures 6(a)-(d) show the influences of the different parameters on the distribution of nanoparticles concentration. It is seen in Figures 6(a)-(c) the nanoparticles concentration decreases with the increase of $W_{e}, M$ and $N_{t}$. Figure $6(d)$ portrayed that the nanoparticles concentration decreases with the increase of $N_{b}$.

\section{Conclusions}

In this investigation, which behavior like a mathematical model describing the heat and mass transfer of blood flow as a Carreau nanofluid with the peristaltic flow conveying gold nanoparticles in an asymmetric channel. The coupled nonlinear governing equations have been modeled and then simplified using long wavelength approximations. The governing equations of motion are analytically solved through Ms-DTM to obtain the distribution of velocity, temperature and concentration. Ms-DTM is applicable to nonlinear models such as Carreau nanofluid models which is more complicated and has a higher degree of non-linearity, in a direct way without using linearization or restrictive assumptions. The main outcomes of the present study are concisely summarized as:

- Thermophoresis and Brownian motion parameters have an opposite effects on pressure gradient and pressure rise. 


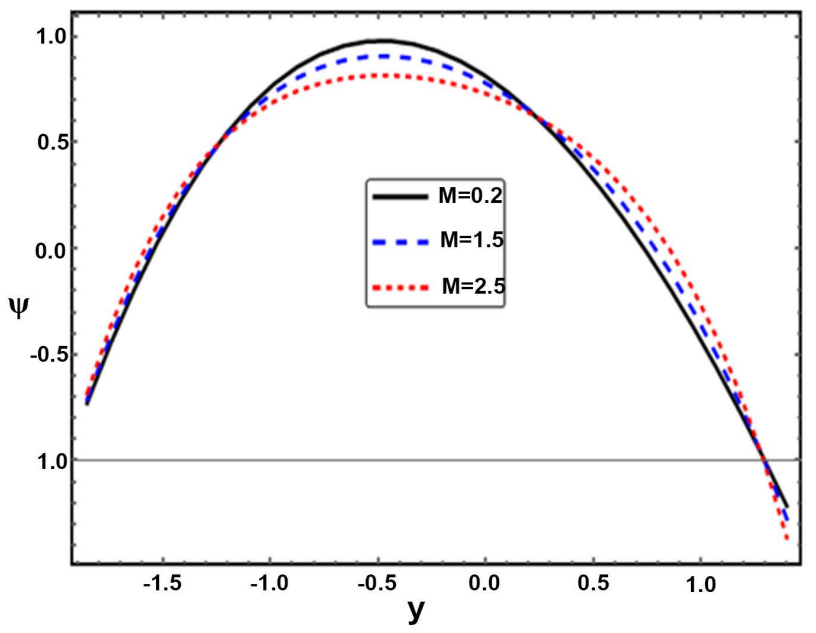

(a)

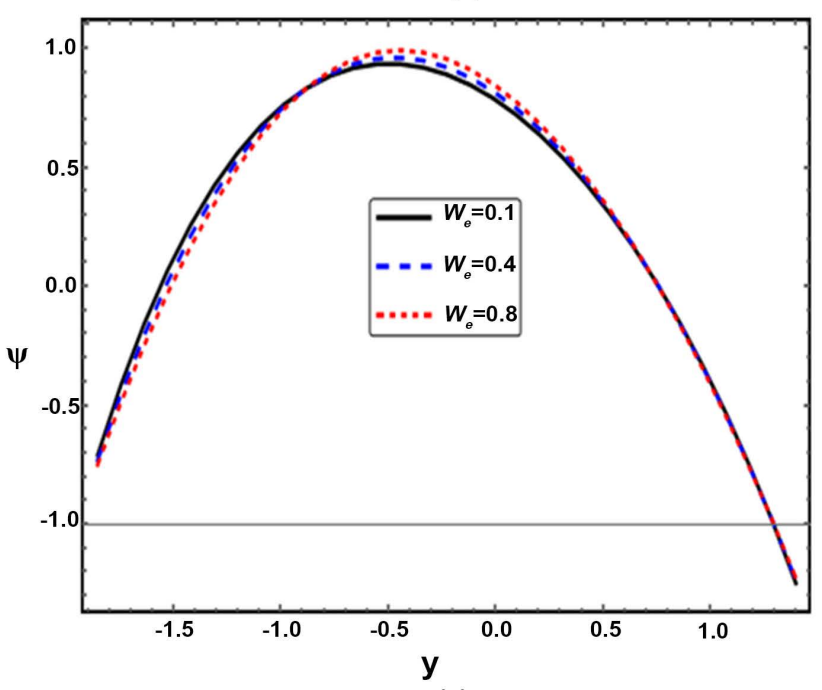

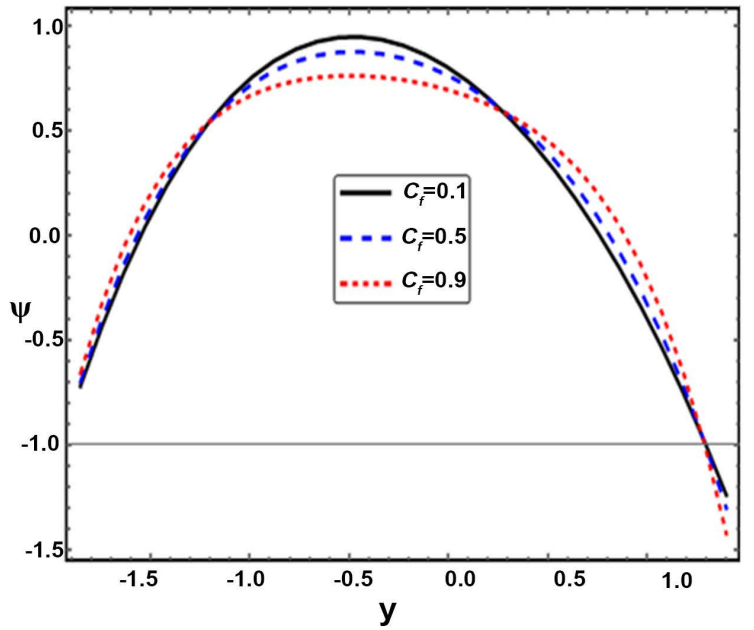

(b)

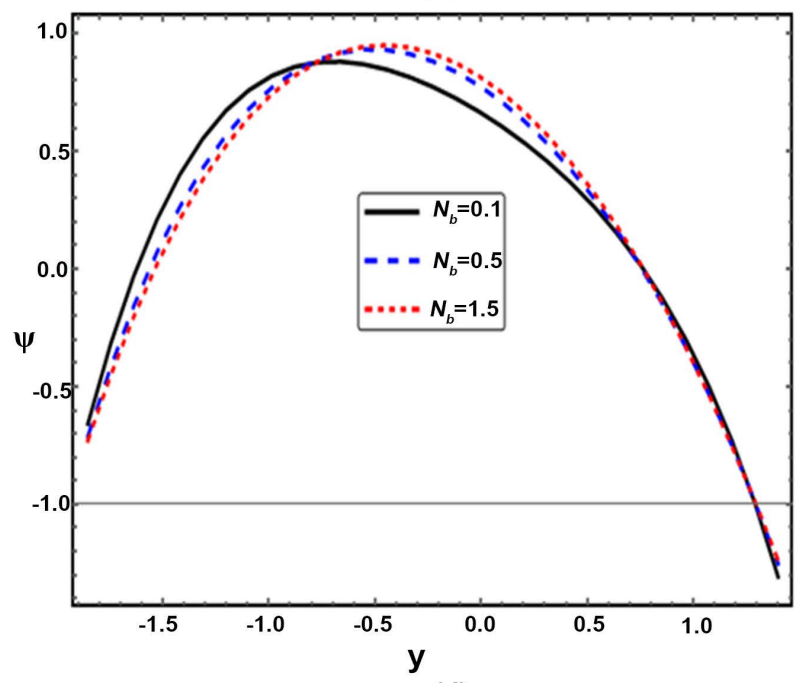

(d)

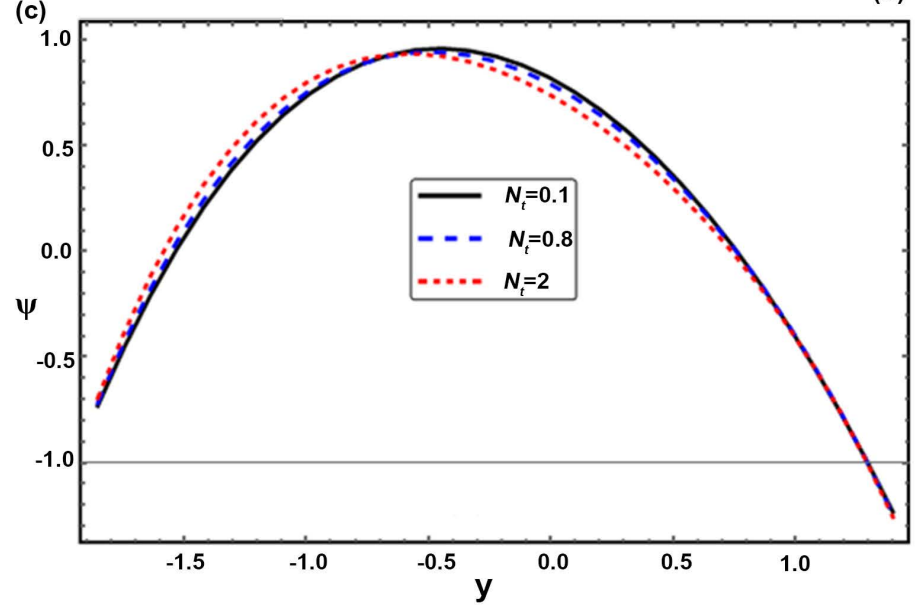

(e)

Figure 4. (a)-(e). Effects of embedded parameters on the velocity profile, (a) $W_{e}=0.5, C_{f}=0.5, N_{t}=0.8, N_{b}=0.7$; (b) $M=1$, $W_{e}=0.5, N_{t}=0.8, N_{b}=0.7$; (c) $M=1, C_{f}=0.5, N_{t}=0.8, N_{b}=0.7$; (d) $M=1, W_{e}=0.5, C_{f}=0.5, N_{t}=0.8$; (e) $M=1, W_{e}=0.5, C_{f}=0.5, N_{b}=0.7$, the other parameters are chosen as $x=0, P_{r}=2, q=1, \vartheta=\frac{\pi}{4}, a=0.4, b=0.5$, $d=1.5, \quad B_{r}=1, n=1, E_{c}=0.2, m=1, \epsilon=0.1, \alpha=0.2, G_{r}=1, L=0.1$. 

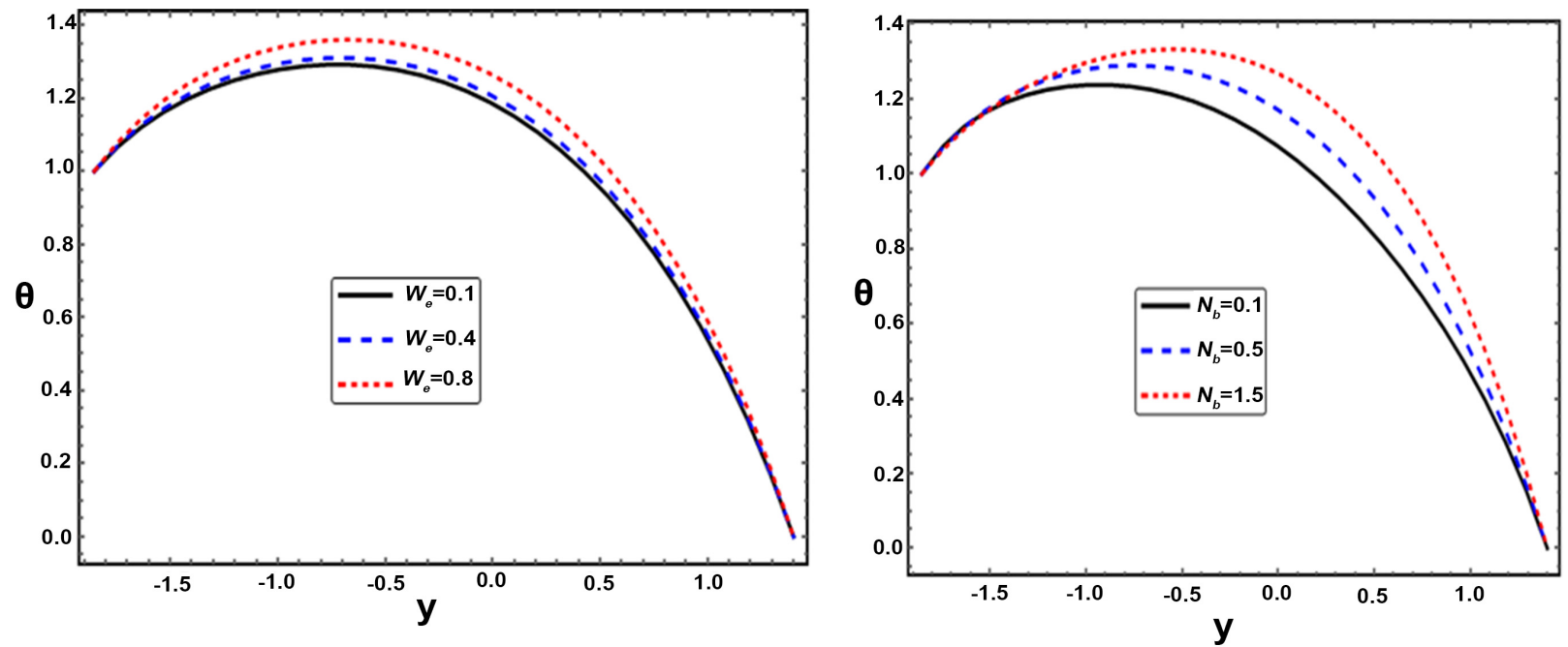

(a)
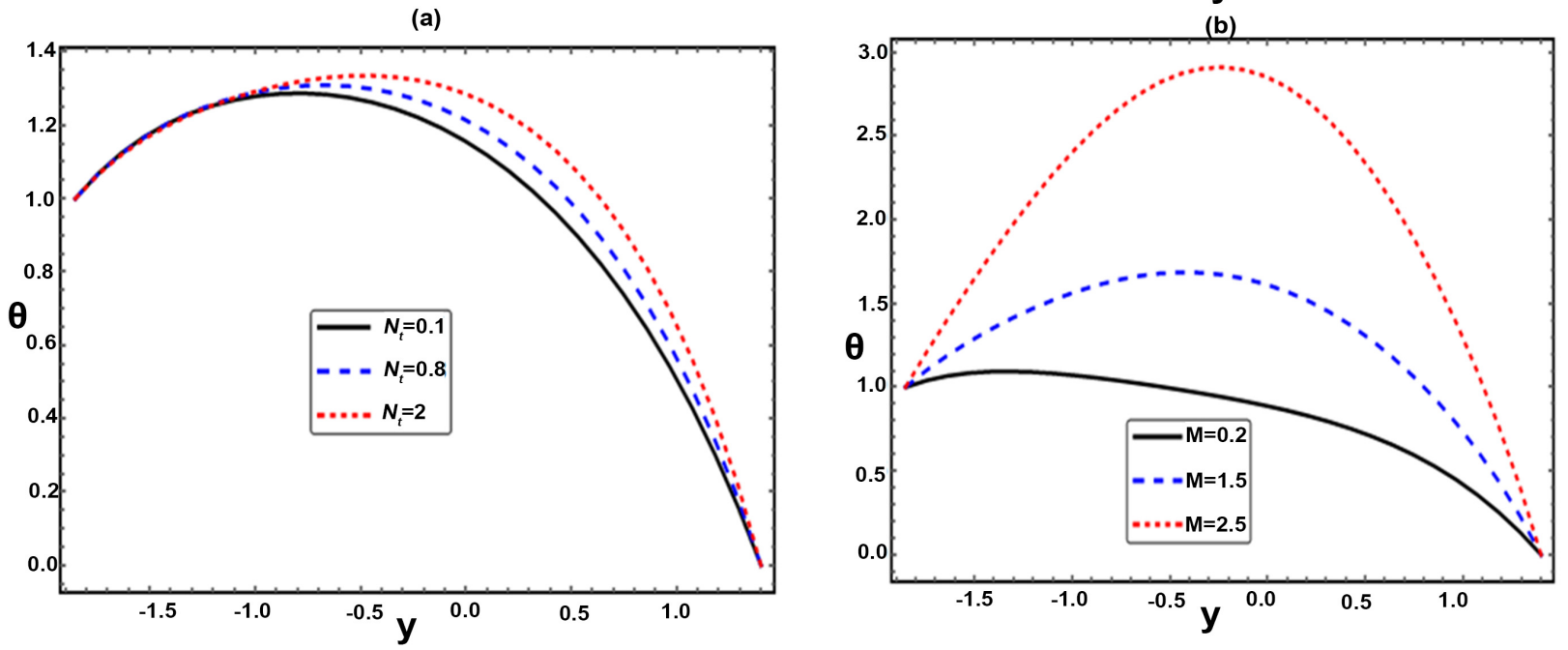

(c)

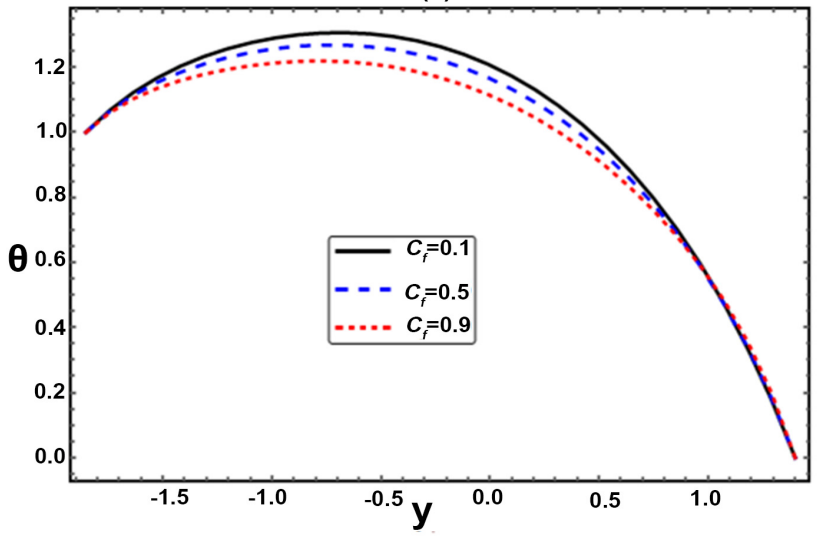

(d)

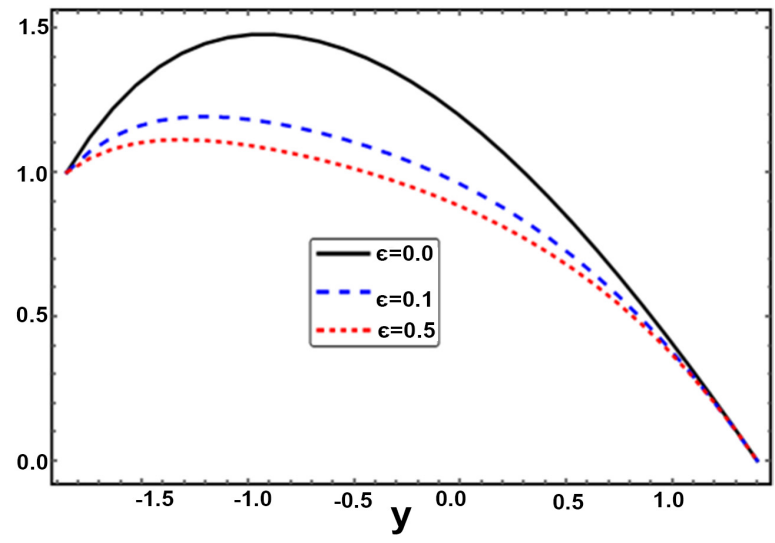

(f)

Figure 5. (a)-(f). Effects of embedded parameters on the temperature profile, (a) $M=1, \epsilon=0.1, C_{f}=0.5, N_{t}=0.8$, $N_{b}=0.7$; (b) $M=1, \epsilon=0.1, W_{e}=0.5, C_{f}=0.5, N_{t}=0.8 ;$ (c) $M=1, \epsilon=0.1, W_{e}=0.5, C_{f}=0.5, N_{b}=0.7 ;$ (d) $\epsilon=0.1, W_{e}=0.5, C_{f}=0.5, N_{t}=0.8, N_{b}=0.7$; (e) $M=1, \epsilon=0.1, W_{e}=0.5, N_{t}=0.8, N_{b}=0.7 ;(\mathrm{f}) \quad M=1, W_{e}=0.5$, $C_{f}=0.5, N_{t}=0.8, N_{b}=0.7$, the other parameters are chosen as $x=0, P_{r}=2, q=1, \vartheta=\frac{\pi}{4}, a=0.4, b=0.5, d=1.5$, $B_{r}=1, n=1, E_{c}=0.2, m=1, \alpha=0.2, G_{r}=1, L=0.1$. 


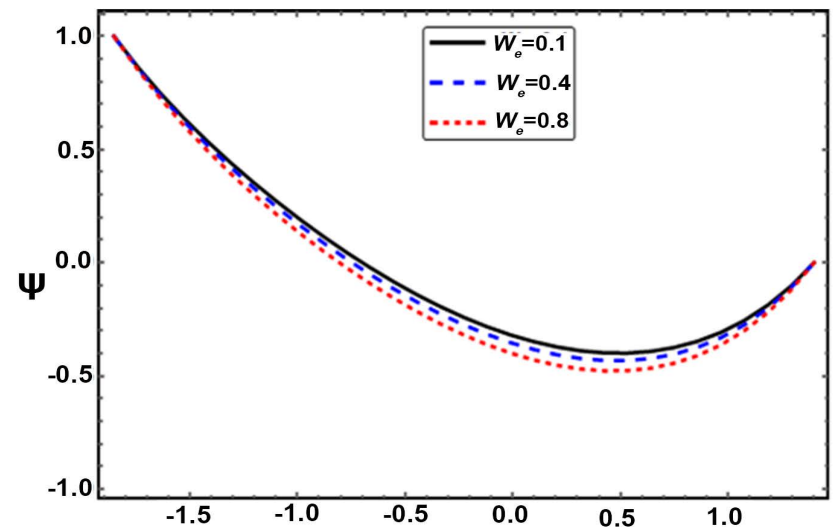

(a)

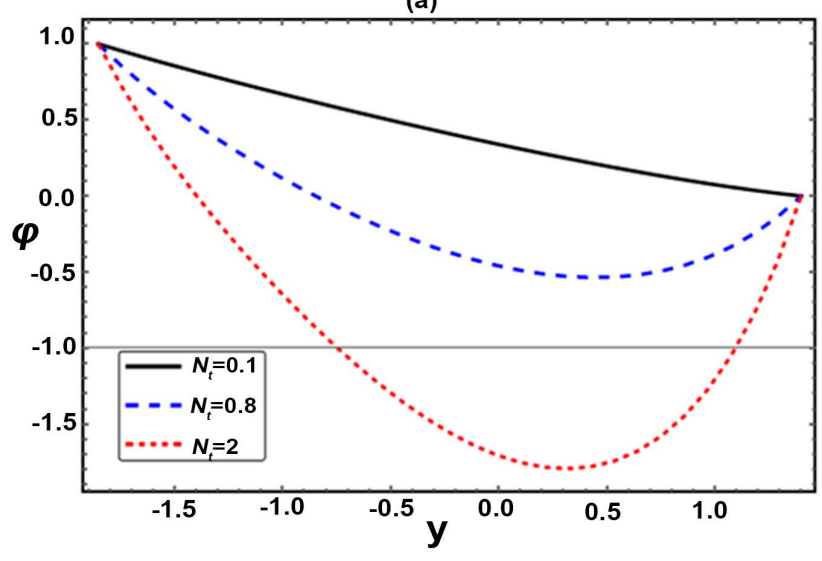

(c)

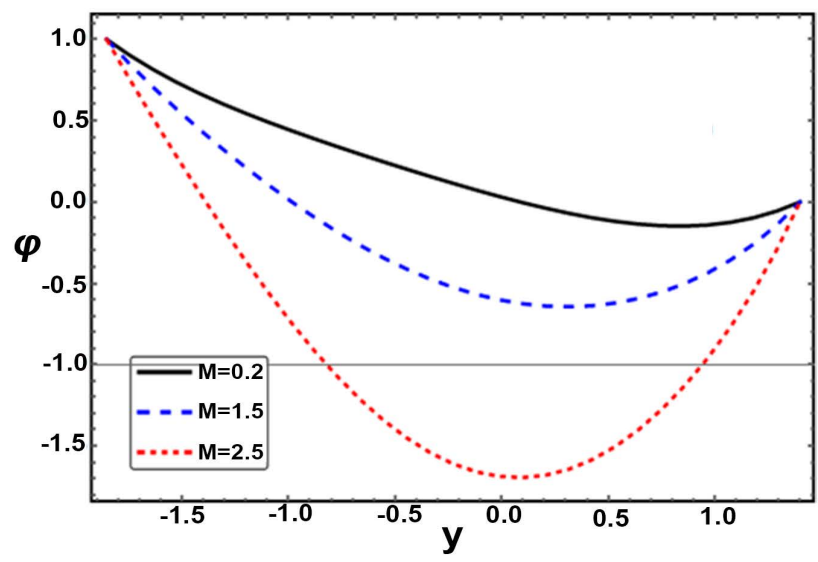

(b)

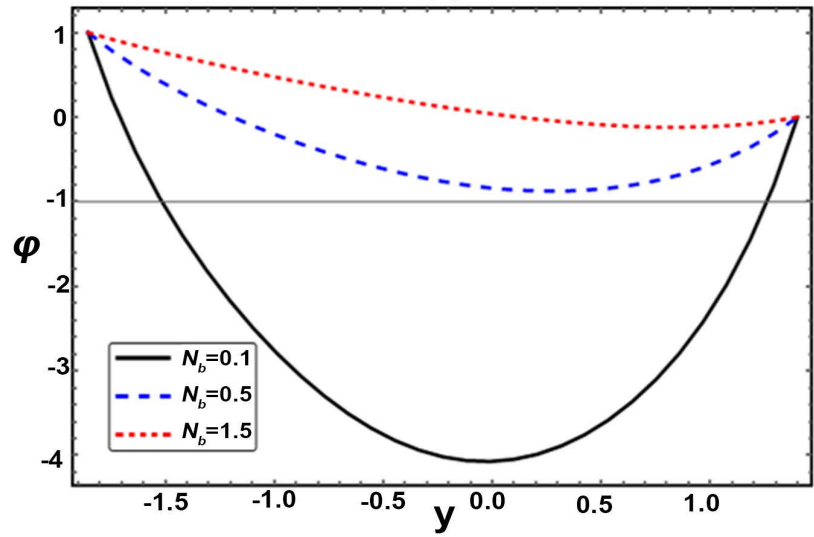

(d)

Figure 6. (a)-(f). Effects of embedded parameters on the concentration profile, (a) $M=1, \epsilon=0.1, C_{f}=0.5, N_{t}=0.8$, $N_{b}=0.7$; (b) $M=1, \epsilon=0.1, W_{e}=0.5, C_{f}=0.5, N_{t}=0.8 ;$ (c) $M=1, \epsilon=0.1, W_{e}=0.5, C_{f}=0.5, N_{b}=0.7 ;(\mathrm{d})$ $\epsilon=0.1, W_{e}=0.5, C_{f}=0.5, N_{t}=0.8, N_{b}=0.7$; (e) $M=1, \epsilon=0.1, W_{e}=0.5, N_{t}=0.8, N_{b}=0.7 ;(\mathrm{f}) \quad M=1, W_{e}=0.5$, $C_{f}=0.5, N_{t}=0.8, N_{b}=0.7$, the other parameters are chosen as $x=0, P_{r}=2, q=1, \vartheta=\frac{\pi}{4}, a=0.4, b=0.5, d=1.5$, $B_{r}=1, n=1, E_{c}=0.2, m=1, \alpha=0.2, G_{r}=1, L=0.1$.

- The behavior of Weissenberg and Forchheimer number on velocity profile are too similar.

- The behavior of pressure gradient with the increase of local Grash of number appears as the dual role phenomenon.

- The gold particles produce the heat which is helpful for treatment of the tumor glands, because it possesses a large atomic number.

- Increases in $N_{t}$ and $N_{b}$ cause to increase in the temperature profile.

- On the nanoparticles concentration the behavior of increasing in $N_{t}$ and $M$ are similar.

Excellent agreement are founded between our results and published results by Akbar et al. [8] through Table 1.

\section{Conflicts of Interest}

The authors declare no conflicts of interest regarding the publication of this paper. 
Table 1. Comparison of velocity, temperature and concentration solutions with exacting published paper Akbar, et al. [8] at the same value of parameter and by taking $C_{f}=0, \epsilon=0, L=0$ and $M=0$.

\begin{tabular}{ccccccccccc}
\hline$y$ & $u(y) N D$ & $u(y)$ & \multirow{2}{*}{ Error } & $\theta(y) N D$ & $\theta(y)$ & Error & \multicolumn{2}{c}{$\varphi(y)$ ND } & \multicolumn{1}{c}{$\varphi(y)$} & Error \\
& Solve & Ms-DTM & & Solve & Ms-DTM & & Solve & Ms-DTM & ETM \\
\hline-1.85355 & -1 & -1 & 0 & 1 & 1 & $1 \times 10^{-8}$ & 1 & 1 & 0 \\
-1.5282 & -0.08505 & -0.08505 & $1 \times 10^{-8}$ & 1.94023 & 1.94023 & $4 \times 10^{-8}$ & -0.920409 & -0.920409 & $2 \times 10^{-8}$ \\
-1.20284 & 0.464817 & 0.464817 & $4 \times 10^{-9}$ & 2.56321 & 2.56321 & $9 \times 10^{-8}$ & -2.28562 & -2.28562 & $7 \times 10^{-8}$ \\
-0.877487 & 0.74798 & 0.74798 & $5 \times 10^{-9}$ & 3.00065 & 3.00065 & $1 \times 10^{-7}$ & -3.32613 & -3.32613 & $1 \times 10^{-7}$ \\
-0.552132 & 0.848036 & 0.848036 & $6 \times 10^{-9}$ & 3.26138 & 3.26138 & $1 \times 10^{-7}$ & -4.05741 & -4.05741 & $2 \times 10^{-7}$ \\
-0.226777 & 0.823794 & 0.823794 & $3 \times 10^{-8}$ & 3.32328 & 3.32328 & $1 \times 10^{-7}$ & -4.44074 & -4.44074 & $2 \times 10^{-7}$ \\
0.0985786 & 0.70687 & 0.70687 & $8 \times 10^{-8}$ & 3.16658 & 3.16658 & $1 \times 10^{-7}$ & -4.44151 & -4.44151 & $3 \times 10^{-7}$ \\
0.423934 & 0.501992 & 0.501992 & $1 \times 10^{-7}$ & 2.78011 & 2.78011 & $1 \times 10^{-7}$ & -4.0402 & -4.0402 & $3 \times 10^{-7}$ \\
0.749289 & 0.187037 & 0.187037 & $2 \times 10^{-7}$ & 2.15477 & 2.15477 & $1 \times 10^{-7}$ & -3.22084 & -3.22084 & $3 \times 10^{-7}$ \\
1.07464 & -0.28777 & -0.28769 & $4 \times 10^{-7}$ & 1.26227 & 1.26277 & $1 \times 10^{-7}$ & -1.93397 & -1.93397 & $2 \times 10^{-7}$ \\
1.4 & -1 & -1 & $6 \times 10^{-7}$ & $6 \times 10^{-7}$ & $3 \times 10^{-7}$ & $2 \times 10^{-7}$ & $1 \times 10^{-6}$ & $1 \times 10^{-6}$ & $4 \times 10^{-7}$ \\
\hline
\end{tabular}

\section{References}

[1] Khan, A.K., Rashid, R., Murtaza, G. and Zahra, A. (2014) Gold Nanoparticles: Synthesis and Applications in Drug Delivery. Tropical Journal of Pharmaceutical Research, 13, 1169-1177. https://doi.org/10.4314/tjpr.v13i7.23

[2] Huang, X. and El-Sayed, M.A. (2010) Gold Nanoparticles: Optical Properties and Implementations in Cancer Diagnosis and Photothermal Therapy. Journal of Advanced Research, 1, 13-28. https://doi.org/10.1016/j.jare.2010.02.002

[3] Hamzehnezhad, A., Fakour, M., Ganji, D.D. and Rahbari, A. (2017) Heat Transfer and Fluid Flow of Blood Flow Containing Nanoparticles through Porous Blood Vessels with Magnetic Field. Mathematical Biosciences, 283, 38-47. https://doi.org/10.1016/j.mbs.2016.11.009

[4] Hatami, M., Hatami, J. and Ganji, D.D. (2014) Computer Simulation of MHD Blood Conveying Gold Nanoparticles as a Third Grade Non-Newtonian Nanofluid in a Hollow Porous Vessel. Computer Methods and Programs in Biomedicine, 113, 632-641. https://doi.org/10.1016/j.cmpb.2013.11.001

[5] Eldabe, N.T. and Abu Zeid, M. (2014) Magnetohydrodynamic Flow with Heat and Mass Transfer of Non-Newtonian Fluid Past a Vertical Heated Plate Embedded in Non-Darcy Porous Medium with Variable Porosity. Innovative Systems Design and Engineering, 5, 60-74.

[6] Rathod, V.P. and Sanjeevkumar, D. (2015) Effect of General Boundary Conditions on the Peristaltic Flow of a Nano Fluid in an Asymmetric Channel Embedded with a Non-Darcy Porous Medium. Journal of Chemical, Biological and Physical Sciences, 6, 72-95.

[7] Wu, Y.S., Bitao, L., Jennifer, L. and Miskimins, M. (2011) Analysis of Multiphase Non-Darcy Flow in Porous Media. Transport Porous Medium, 88, 205-223. https://doi.org/10.1007/s11242-011-9735-8

[8] Akbar, N.S., Nadeem, S. and Khan, Z.H. (2014) Numerical Simulation of Peristaltic Flow of a Carreau Nanofluid in an Asymmetric Channel. Alexandria Engineering Journal, 53, 191-197. https://doi.org/10.1016/j.aej.2013.10.003

[9] Akram, S. (2014) Effects of Nanofluid on Peristaltic Flow of a Carreau Fluid Model in an Inclined Magnetic Field. Heat Transfer-Asian Research, 43, 368-383. 
https://doi.org/10.1002/htj.21082

[10] Eldabe, N.T.M., Abo-Seida, O.M., Abo-Seliem, A.S., ElShekhipy, A.A. and Hegazy, N. (2017) Peristaltic Transport of Magnetohydrodynamic Carreau Nanofluid with Heat and Mass Transfer inside Asymmetric Channel. American Journal of Computational Mathematics, 7, 1-20. https://doi.org/10.4236/ajcm.2017.71001

[11] Nowar, K. (2014) Peristaltic Flow of a Nanofluid under the Effect of Hall Current and Porous Medium. Mathematical Problems in Engineering, 2014, Article ID: 389581. https://doi.org/10.1155/2014/389581

[12] Abo-Eldahab, E., Barakat, E. and Nowar, K. (2012) Hall Currents and Heat Transfer Effects on Peristaltic Transport in a Vertical Asymmetric Channel through a Porous Medium. Mathematical Problems in Engineering, 2012, Article ID: 840203. https://doi.org/10.1155/2012/840203

[13] Seth, G.S. and Ghosh, S.K. (1986) Combined Influence of Rotation and Hall Current in the Presence of a Transverse Magnetic Field. Indian Journal of Pure and Applied Mathematics, 17, 819-826.

[14] Ghosh, S.K. (1999) Hall Effect on Unsteady Hydromagnetic Flow in a Rotating Channel Permeated by an Inclined Magnetic Field in the Presence of an Oscillator. Czechoslovak Journal of Physics, 49, 465-472. https://doi.org/10.1023/A:1022812824266

[15] Mekheimer, K.S. and Abd Elmaboud, Y. (2014) Simultaneous Effects of Variable Viscosity and Thermal Conductivity on Peristaltic Flow in a Vertical Asymmetric Channel. Canadian Journal of Physics, 92, 1541-1555. https://doi.org/10.1139/cjp-2013-0465

[16] Thakur, P.M. and Hazarika, G.C. (2014) Effects of Variable Viscosity and Thermal Conductivity on Flow and Heat Transfer over a Stretching Surface with Variable Heat Flux in Micropolar Fluid in Presence Magnetic Field. International Journal of Scientific and Innovative Mathematical Research, 2, 554-566.

[17] Zhou, J.K. (1986) Differential Transformation and Its Applications for Electrical Circuits. Huazhong University Press, Wuhan, China.

[18] Erturk, V., Momani, S. and Odibat, Z. (2008) Application of Generalized Differential Transform Method to Multi-Order Fractional Differential Equations. Communication Nonlinear Science and Numerical Simulation, 13, 1642-1654. https://doi.org/10.1016/j.cnsns.2007.02.006

[19] Odibata, Z.M., Bertelle, C., Aziz-Alaoui, M.A. and Duchamp, G.H.E. (2010) A Multi-Step Differential Transform Method and Application to Non-Chaotic or Chaotic Systems. Computers and Mathematics with Applications, 59, 1462-1472. https://doi.org/10.1016/j.camwa.2009.11.005

[20] Bharali, A. and Borkakati, A.K. (1982) The Effect of Hall Currents on MHD Flow and Heat Transfer between Two Parallel Porous Plates. Applied Scientific Research, 39, 155-165. https://doi.org/10.1007/BF00457017

[21] Akbar, N.S., Nadeem, S., Hayat, T. and Hendi, A.A. (2012) Peristaltic Flow of a Nanofluid with Slip Effects. Meccanica, 47, 1283-1294.

https://doi.org/10.1007/s11012-011-9512-3 